\title{
Gyrosheath Near the Tokamak Edge
}

\author{
R.D. Hazeltine, H. Xiao \\ Institute for Fusion Studies \\ The University of Texas at Austin \\ Austin, Texas 78712 \\ and \\ P.M. Valanju \\ Fusion Research Center \\ The University of Texas at Austin \\ Austin, Texas 78712 \\ DOE/ET/53088--599 \\ DE؟ 3012191
}

\begin{abstract}
A new model for the structure of the radial electric field profile in the edge during the $\mathrm{H}$-mode is proposed. Charge separation caused by the difference between electron and ion gyromotion, or more importantly in a tokamak, the banana motion (halo effect) can self-consistently produce an electric dipole moment that causes the sheared radial electric field. The calculated results based on the model are consistent with D-III D and TEXTOR experimental results.
\end{abstract}

Pacs Nos.: $52.55,52.25,52.20$ 


\section{Introduction}

After its first observation on $\mathrm{ASDEX},{ }^{1}$ the transition from low confinement (L-mode) to high confinement ( $\mathrm{H}$-mode) has been observed in many tokamaks ${ }^{2-5}$ with different heating and operational schemes. ${ }^{6-8}$ The improvement of energy as well as particle confinement time is the most important character of the $\mathrm{H}$-mode. Other properties of $\mathrm{H}$-mode include a rapid increase of electron density, a sudden drop of $H_{\alpha} / D_{\alpha}$ recycling, and sudden appearance of radial electric field near the plasma periphery. The $\mathrm{L}-\mathrm{H}$ transition can also be triggered by an external electric field imposed by a biased electrostatic probe, with either positive $\left(\mathrm{H}_{+}\right.$ mode) or negative ( $H_{-}$mode) voltages. ${ }^{9}$

In recent D-III D and TEXTOR experiments, ${ }^{10,11}$ a kink in electron density profile near the last closed flux surface is observed. We propose that this kink is formed due to the fast electron loss to the scrape-off layer. The resulting space charge is the source of the radial electric field, which is widely believed to play a crucial role in L-H transition for suppressing turbulence. ${ }^{12}$ Because ion gyromotion (or the banana motion) forbids a kink in its density profile, the charge separation near the kink, within the scale length of ion (poloidal) gyroradius, becomes inevitable when the electron kink forms. This is the so called halo effect. The resulting sheared electric field makes the charged plasma rotate, ${ }^{13}$ causing shear flow.

\section{Density Broadening}

If $f_{g c}$ is the distribution of guiding centers, then the charge density at position $\mathbf{x}$ is given by:

$$
n(\mathbf{x})=\int d^{3} v f_{g c}(\mathbf{x}-\boldsymbol{\rho}(\mathbf{v}), \mathbf{v})
$$


where $\boldsymbol{\rho} \equiv \mathbf{b} \times \mathbf{v} / \Omega$ is the vector gyroradius, $\mathbf{b}=\mathbf{B} /|\mathbf{B}|$ is the unit vector of magnetic field, and $\Omega=e B / m c$ is the gyrofrequency.

Suppose that there is symmetry in the $y$ and $z$ directions and that $\mathbf{b}=\mathbf{z}$, so that

$$
\rho_{x}=-\frac{v_{y}}{\Omega}, \quad \rho_{y}=\frac{v_{x}}{\Omega}, \quad \rho_{z}=0
$$

and

$$
f_{g c}(\mathbf{x}-\boldsymbol{\rho}(\mathbf{v}) \mathbf{v})=f_{g c}\left(x+\frac{v_{y}}{\Omega} \mathbf{v}\right)
$$

Further we suppose that

$$
f_{y c}(x, \mathbf{v})=\frac{n_{g c}(x)}{n_{M}} f_{M}(\mathbf{v})
$$

where $f_{M}$ is a Maxwellian with constant density $n_{M}$. We introduce thermal velocity $v_{t h}=$ $(2 T / m)^{1 / 2}, s=v_{y} / v_{t h}, \rho=v_{t h} / \Omega$ and integrate over $v_{x}$ and $v_{z}$ to express the density as

$$
n(x)=\frac{1}{\sqrt{\pi}} \int d s e^{-s^{2}} n_{g c}(x+s \rho)
$$

Equation (2) shows the broadening of the density in its general form. For concreteness, we assume that $n_{g c}$ is Gaussian:

$$
n_{g c}(x)=\frac{1}{d} \exp \left(-\frac{x^{2}}{d^{2}}\right)
$$

Then Eq. (2) gives

$$
n(x)=\frac{1}{\sqrt{d^{2}+\rho^{2}}} \exp \left(-\frac{x^{2}}{d^{2}+\rho^{2}}\right) .
$$

It can be seen that $n(x)$ is a broadened version of $n_{g c}$. In a simple description, because of the rapid gyromotion an ion can be considered as smeared over its gyroradius, so that its average density can't change faster than the gyroradius. The value and slope of $n(x)$ is not affected by the smoothing, only the second derivative (curvature) is reduced. When $n_{e}(x) \approx n_{g c}(x)$ is broader than ion gyroradius $\rho_{i}, n_{i}(x)$ can match $n_{e}(x)$ to persevere quasineutrality. But when $n_{e}$ has curvature radius $r_{n}=\left[n_{e}(r) /\left(d^{2} n_{e} / d r^{2}\right)\right]^{1 / 2}$ shorter than $\rho_{i}$, local quasi-neutrality becomes impossible. 


\section{Simple Model for Gyrosheath}

We write Poisson's equation as:

$$
\frac{\lambda_{D}^{2}}{\rho^{2}} \phi^{\prime \prime}=g(r)-\mathbf{S} * f(r)
$$

where $\phi=e \Phi / T$ is the normalized electrostatic potential, $\lambda_{D}=\left(T / 4 \pi n_{0} e^{2}\right)^{1 / 2}$ is the Debye length of the plasma, $g(r)$ and $f(r)$ are the electron density and ion guiding center densities respectively (both normalized to $n_{0}$ ), $r$ is the radial length normalized by Larmor radius $\rho$, and $\mathbf{S}$ is the gyro-smoothing operator:

$$
\mathbf{S} * f(r)=\frac{1}{\sqrt{\pi}} \int_{-\infty}^{\infty} d s e^{-s^{2}} f(r+s)
$$

where $\rho$ is the ion gyroradius.

Keeping in mind the DIII-D and TEXTOR results, we choose a simple model for the electron density profile near the kink region:

$$
g(r)= \begin{cases}m_{1} r+g_{0} & \text { if } r<0 \\ m_{2} r+g_{0} & \text { if } r>0\end{cases}
$$

where $m_{1} \neq m_{2}$ are the slopes on both sides of the kink, $g_{0}$ is the normalized density at the kink. On both sides of the kink, if $|r|>(2-3) \rho_{p}$, plasma should be quasi-neutral, so that $(\mathbf{S} * f-g)$ should equal zero. The TEXTOR data shows that on both sides the plasma potential $\phi$ is nearly constant, so that the monopole moment in this boundary layer must be very small $(\approx 0)$. If there are multipoles in the charge separation region, the radial electric field due to them will be vary large, and the perpendicular conductivity $\sigma_{\perp}=\nu m_{i} / n_{i} e^{2}$ will quickly damp them to zero. However, the dipole moment can't become zero due to the gyro-smoothing operator.

To see how the smoothing operator suppressed all moments higher than the dipole, we express the ion guiding center density near the kink as a Fourier series,

$$
f=\sum_{n=-\infty}^{\infty} A_{n} e^{i n x}
$$


where $x$ is normalized with poloidal ion gyroradius. Acting with the smoothing operator, we find

$$
F=\mathbf{S} * f=\sum_{n=-\infty}^{\infty} A_{n} e^{i n x} e^{-n^{2} / 4}
$$

The even terms of the series should vanish to maintain total charge neutrality in the tokamak plasma, while the amplitudes of the odd terms beyond $n=1$ are strongly suppressed, so that the only difference between $g$ and $F$ is a dipole moment $(n=1)$. We therefore choose the ion guiding center density as:

$$
f(r)= \begin{cases}m_{1} r+g_{0} & \text { if } r<\Delta \\ m_{2} r+g_{0} & \text { if } r>\Delta\end{cases}
$$

where $\Delta$ is a shift distance of order one. ${ }^{14}$

After the integration the smoothed ion density profile becomes:

$$
\begin{aligned}
\mathbf{S} * f(r) & =g_{0}+r \frac{m_{1}+m_{2}}{2}+r \frac{m_{1}-m_{2}}{2} \operatorname{erf}(\Delta-r) \\
& +\frac{m_{2}-m_{1}}{2 \sqrt{\pi}} e^{-(\Delta-r)^{2}}
\end{aligned}
$$

where $\operatorname{erf}(x)$ is the error function:

$$
\operatorname{erf}(x)=\frac{2}{\sqrt{\pi}} \int_{0}^{x} d s e^{-s^{2}}
$$

Then we can calculate the charge separation, electrostatic potential and radial electric field.

\section{Trapped Particle Effect}

In a tokamak plasma, trapped particles have the largest orbits. Since the bounce time of a trapped particle $\tau_{b} \approx R / v_{\|}$is much shorter than the diffusion time $\tau_{D} \approx a^{2} / D$, where $D$ is the diffusion coefficient, the characteristic length of the trapped ion density can't be smaller than the ion banana width, $\Delta_{b} \approx \sqrt{\epsilon} \rho_{p}$. In other words, trapped particle orbits lead to a ion charge density smoothing, similar to gyro-smoothing, except that the characteristic smoothing length is $\Delta_{b}$, not $\rho$. 
In a tokamak, the charge separation arises mainly from trapped particles, and the electric field due to the charge separation itself changes the trapped population. Thus the radial electric field should be determined by the balance of the two effects.

From Poisson's equation and the above argument, the radial electric field due to the charge separation can be written as:

$$
E_{r}=4 \pi n_{0} e \eta_{t}\left(E_{r}\right) \sqrt{\epsilon} \rho_{p} \int\left(\mathbf{S} * f\left(r^{\prime}\right)-g\left(r^{\prime}\right)\right) d r^{\prime}
$$

where $\eta_{t}\left(E_{r}\right)$ is the fraction of the trapped particle due to the radial electric field and $r^{\prime}$ is the radial distance normalized by the ion banana width. Because the radial electric field shifts the trapped particles to the Maxwellian tail ${ }^{15}$, approximately we have

$$
\eta_{t}\left(E_{r}\right) \approx \sqrt{\epsilon} e^{-\tilde{V}_{E_{p}}^{2}}
$$

where $\tilde{V}_{E_{p}}=c E_{r} / v_{i} B_{p}$ is the normalized toroidal $\mathbf{E} \times \mathbf{B}$ velocity. Therefore we have

$$
\tilde{V}_{E_{p}} e^{\tilde{V}_{E_{p}}^{2}}=\epsilon \frac{c^{2}}{V_{A_{p}}^{2}} \int(\mathbf{S} * f-g) d r^{\prime}
$$

where $V_{A_{p}}=\left(4 \pi n_{0} m_{i} / B_{p}^{2}\right)^{1 / 2}$ is the poloidal Alfvén speed. We can solve Eq. (10) numerically and calculate the radial electric field.

From DIII-D parameters, we have $c^{2} / V_{A_{p}}^{2} \sim 10^{5}, \epsilon \sim 0.34$, and the integral in Eq. (10) is of order 0.1. The solution of Eq. (10) gives $\tilde{V}_{E_{p}} \sim 2.8$. This is in fair agreement to the experiment result, which gives $\tilde{V}_{E_{p}} \sim 1$. The higher value of electric field we get may be because the actual kink in the electron density is not the infinitely sharp kink we have assumed in our model equation (5) and (6). More importantly, we only considered the electric field effect on the trapped population, and ignored the electric field effect on the particle motion. We expect to reduce $\tilde{V}_{E_{p}}$ if we take both these effects into account. 


\section{Discussion and Summary}

The electron density profile is found to have a kink near the separatrix $(R \approx 2.28 \mathrm{~m})$ during the $\mathrm{L}-\mathrm{H}$ transition in the DIII-D experiment. ${ }^{10}$ At that point, the electron density curvature radius is smaller than the ion poloidal gyroradius, which is about $1 \mathrm{~cm}$. Thus it is impossible for the ion density to follow the electron density to maintain local quasi-neutrality.

The results of the calculations of the charge separation, radial electric field, and electrostatic potential are shown in Fig. 1. We find that the model worked very well in this case in which electron density has a shoulder-like kink profile, compared with the D-III D experiment results shown in Fig. 2 of Ref. 10. Also during VH-mode discharge, it is observed that the density kink and shear layer move inside at the same time. ${ }^{16,17}$ This supports our model.

In recent $\mathrm{TEXTOR}^{11}$ experiments, radial electric fields have been imposed on the plasma edge by means of a biased electrode to investigate the influence on the plasma edge profiles and its confinement. Above a critical bias potential a kink appears in the electron density profile near the plasma edge $(r \approx 46 \mathrm{~cm})$, with a curvature radius much smaller than the ion poloidal gyroradius. Using this electron density profile in our model, we can get the dipole charge separation and the sheared electric field. We find that the calculated electric potential in Fig. 2 agrees well with the experimental measurement as shown in Fig. 9 of Ref. 11. The only difference is a small shift in the radial position. This is because our model fixes the dipole moment value but not its position to within an ion (poloidal) gyroradius.

In HL-1 tokamak ${ }^{18}$ different isotopes (hydrogen and deuterium) have been used in bias electrode induced $\mathrm{H}$-mode experiments. The results show that it is easier to trigger $\mathrm{H}$-mode in D-plasma than that in H-plasma. This seems to support our model because the banana width of deuterium is larger than that of hydrogen.

In summary, a new model for the structure of the radial electric field in the edge during the 
H-mode has been proposed. The major idea is that the ion density profile can't sustain sharp radial features due to the gyromotion or more importantly, banana motion. The curvature radius of the ion density change can't become smaller than its gyroradius or banana width. When the electron density profile changes so sharply that its changing scale length is smaller than the banana width, the ion density can't follow the change. The resulting dipole charge distribution produces a sheared electric field. Because the radial electric field changes the trapped population, a self-consistent radial electric field can be obtained by the balance of the two effects. The whole process, from charge separation to formation of sheared electric field, is very fast, matching the experimental observation of prompt potential change during L-H transition.

\section{Acknowledgments}

We acknowledge discussions with Dr. P. Yushmanov, Dr. H.C. Ye, and Dr. Z.T. Wang.

Author H. Xiao is grateful for helpful discussions with Dr. S.T. Tsai, Dr. Y.Z. Zhang, and Dr. J.Q. Dong.

This work was supported by the U.S. Department of Energy contract \#DE-FG05-80ET5.3088. 


\section{References}

1. F. Wagner, G. Becker, K. Behringer, D. Campbell, A. Eberhangen. W. Engelhardt, G. Fussmann, O. Gehre, J. Gernhardt, G. V. Gierke, G. Hass, M. Huang, F. Karger, M. Keilhacker, O. Kluber, M. Kornherr, K. Lackner, G. Lisitano, G.G. Lister, H.M. Mayer, D. Meisel, E.R. Muller, H. Murmann, H. Nledermeyer, W. Poschenrieder, H. Rapp, H. Rohr, F. Schneider, G. Siller, E. Speth, A. Stabler, K. H. Steuer, G. Venus, and O. Vollmer, Phys. Rev. Lett. 49, 1408 (1982).

2. S.M. Kaye, M.G. Bell, K. Bol, D. Boyd, K. Brau, D. Buchenauer, R. Budny, A. Cavallo, P. Couture, T. Crowley, D. S. Darrow, H. Eubank, R. J. Fonck, R. Goldston, B. Grek, K.P. Jaehnig, D. Johnson, R. Kaita, H. Kugel, B. LeBlanc, J. Manicam, D. Manos, D. Mansfield, E. Mazzucato, R. McCann, K. McGuire, D. Mueller, A. Murdock, M. Okabayashi, K. Okano, D. K. Owens, D. E. Post, M. Reusch, G. L. Schmidt, S. Sesnic, R. Slusher, S. Suckewer, C. Surko, H. Takahashi, F. Tenney, H. Towner, and J. Valley, J. Nucl. Mater. 121, 115 (1984).

3. A. Tanga, K.H. Behringer, A.E. Costley, M. Brusati, B. Denne, A. Edwards, A. Gibson, R.D. Gill, N. Gottardi, R. Granetz, P.J. Harbour, H. Jackel, M. Keihacker, E. Lazzaro, M. Malacarne, P.D. Morgan, P. Noll, J. Orourke, P.E. Stott, D.R. Summers, J.A. Tagle, and P.R. Thomas, Nucl. Fusion 27, 1877 (1987).

4. S. Sengoku, A. Finahashi, M. Hasegawa, K. Hoshino, S. Kasai, T. Kawakami, H. Kawashima, T. Maatoba, T. Matsuda, H. Matsumoto, Y. Miura, M. Mori, H. Ogawa, T. Ohtsuka, T. Shoji, N. Suzuki, H. Tamai, Y. Uesugi, T. Yamamoto, and T. Yamauchi, Phys. Rev. Lett. 59, 450 (1987).

5. C.E. Bush, R.J. Goldston, S.D. Scott, E.D. Fredrickson, K. McGuire, J. Schivell, G. 
Taylor, C.W. Barnes, M.G. Bell, R.L. Boivin, N. Bretz, R.V. Budny, A. Cavallo, P.C. Eftthimion, B. Grek, R. Hawryluk, K. Hill, R.A. Hulse, A. Janos, D.W. Johnson, S. K.ilpatrick, D.M. Manos, D.K. Mansfield, D.M. Meade, H. Park, A.T. Ramsey, B. Stratton, E.J. Synakowski, H.H. Towner, R.M. Wieland, M.C. Zarnstorff, and S. Zweben, Phys. Rev. Lett. 65, 424 (1990).

6. T.H. Osborne, N.H. Brooks, K.H. Burrell, T.N. Carlstrom, R.J. Groebner, W. Howl, A.G. Kellman, L.L. Lao, T.S. Taylor, D.N. Hill, N. Ohyabu, and M.E. Perry, Nucl. Fusion 30, 2023 (1990).

7. S. Tsuji, K. Ushigusa, Y. Ikeda, T. Imai, T. Itami, M. Nemoto, K. Nagashima, Y. Koida, Y. Kawano, T. Fukuda, T. Komdoh, M. Shimada, H. Nakamura, O. Naito, H. Yoshida, T. Nishitani, H. Kubo, K. Yoshino, and K. Uehara, Phys. Rev. Lett. 64, $1023(1990)$.

8. B.J.D. Tubbing, B. Balet, D.V. Bartlett, C.D. Challis, S. Corti, R.D. Gill, C. Gormezano, C.W. Gowers, M. von Hellermann, M. Hugon, J.J. Jacquinot, H. Jaeckel, P. Kupschus, K. Lawson, H. Moris, J. O'Rourke, D. Pasini, F.G. Rimini, G. Sadler, G.L. Schmidt, D.F.H. Start, P.M. Stubberfield, A. Tanga, and F. Tibone, Nucl. Fusion 31, 2365 (1989).

9. R.R. Weynants and R.J. Taylor, Nucl. Fusion 30, 945 (1990).

10. E.J. Doyle, C.L. Rettig, K.H. Burrell, P. Gohil, R.J. Groebner, T.K. Kurki-Suonio, R.J. LaHaye, R.A. Moyer, T.H. Osborne, W.A. Peebles, R. Philipona, T.L. Rhodes, T.S. Taylor, and J.G. Watkins, Turbulence and Transport Reduction Mechanisms in the Edge and Interior of DIII-D H-Mode Plasmas, Proc. 14th Inter. Conf. on Plasma Phys. Contr. Nucl. Fusion, Würzburg, Germany, IAEA-CN-56/A-4-1, (1992). 
11. R.R. Weynants, G. Van Oost, G. Bertschinger, J. Boedo, P. Brys, T. Delvigne, K.H. Dippel, F. Durodie, H. Euringer, K.H. Finken, D.S. Gray, J.D. Hey, D.L. Hillis, J.T. Hogan, L. Konen, R. Leners, A.M. Messiaen, A. Pospieszczyck, U. Samm, R.P. Schweer, G. Telesca, R. Van Nieuwenhove, and P.E. Vandenplas Nucl. Fusion 32, 837 (1992).

12. H. Biglari, P.H. Diamond, and P.W. Terry, Phys. Fluids B 2, 1 (1990).

13. R.C. Davidson, Theory of Nonneutral Plasmas, (W.A. Benjamin, Inc., 1974).

14. A similar result has been obtained by solving the gyrokinetic equtaion. J. W. Conner, private communication.

15. H.L. Berk and A.A. Galeev, Fluids Phys. 10, 441 (1967)

16. G.L. Jackson, J. Winter, T.S. Taylor, C.M. Greenfield, K.H. Burrell, T.N. Carlstrom, J.C. DeBoo, E.J. Doyle, R.J. Groebner, L.L. lao, C. Rettig, D.P. Schissel, E.J. Strait, and the DIII-D Research Team, Phys. Fluids B 4, 2181 (1992).

17. K.H. Burrell, P. Gohil, R.J. Groebner, and R.P. Seraydarian, Bull. Am. Phys. Soc. 37, 1410 (1992).

18. Y.Z. Zheng, L.B. Ran, E.Y. Wang, H.R. Yang, X.T. Ding, Z.H. Wang, L.H. Yao, S.Q. Sun, X.D. Li, J.F. Dong, H.C. Deng, Z.C. Deng, G.C. Guo, J.W. Yang, Y.X. Jiang, S.K. Yang, K.H. Li, M.L. Shi, Q.W. Yang, S.X. Zhang, H.N. Zuo, D.F. Gong, C.X. Luo, H.Z. Li, B. Fu, G.W. Zhong, Y.X. Yin, Y. Lui, J.E. Zhen, Y.W. Qin, B.R. Shi, X.W. Deng, and HL-1 Team, Studies of Improved Confinement on HL-1, Proc. 14th Inter. Conf. on Plasma Phys. Contr. Nucl. Fusion, Würzburg, Germany, IAEA-CN56/A-7-10, (1992). 


\section{Figure Captions}

1. Charge separation and radial electric field calculated from gyrosheath model with DIIID parameters. Comparision with Fig. 2 of Ref. 10 shows good agreement.

2. Charge separation and radial profile of electrostatic potential calculated from gyrosheath model with TEXTOR parameters. Comparision with Fig. 9 of Ref. 11 shows good agreement. 


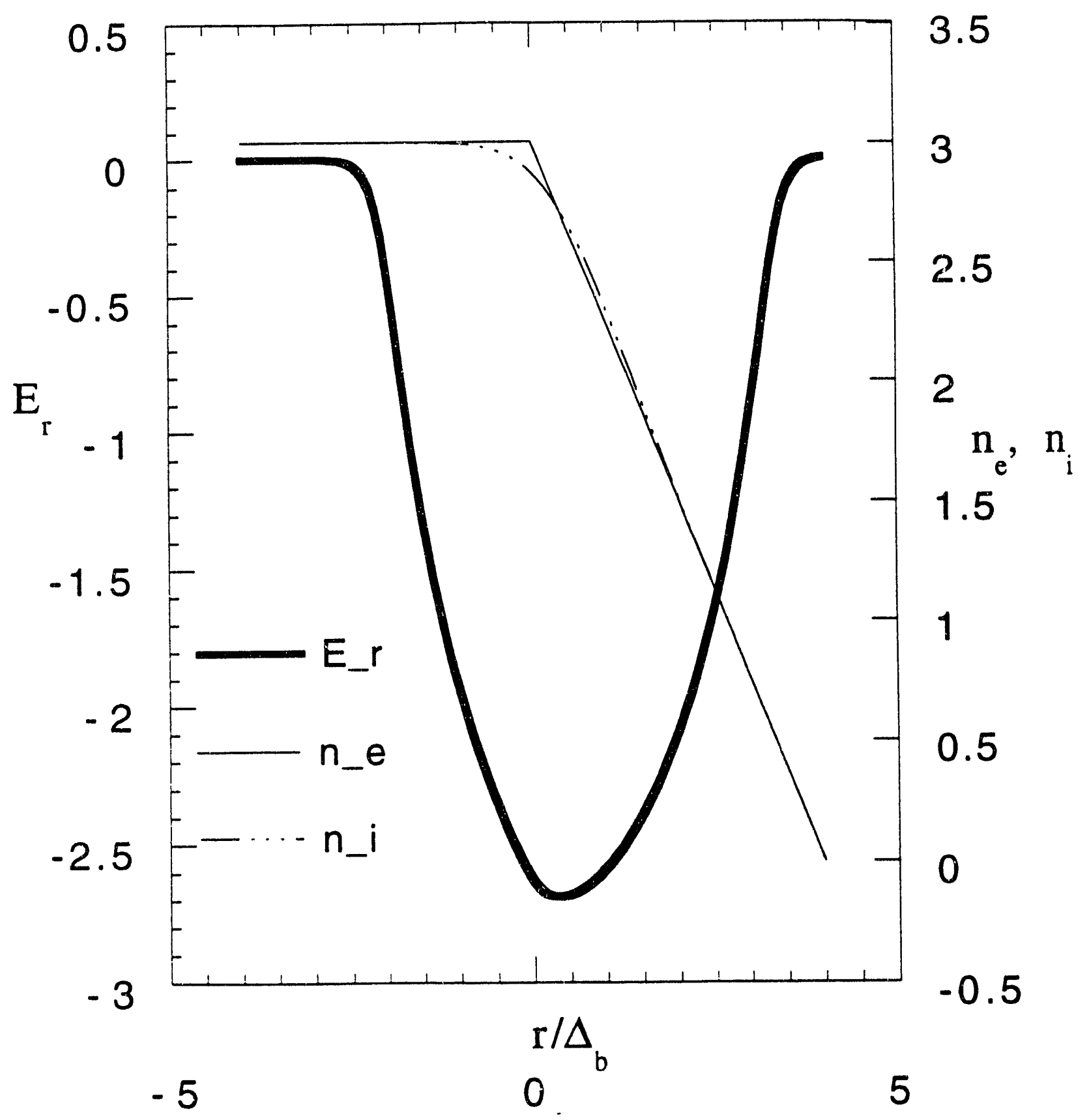

Fig. 1. 


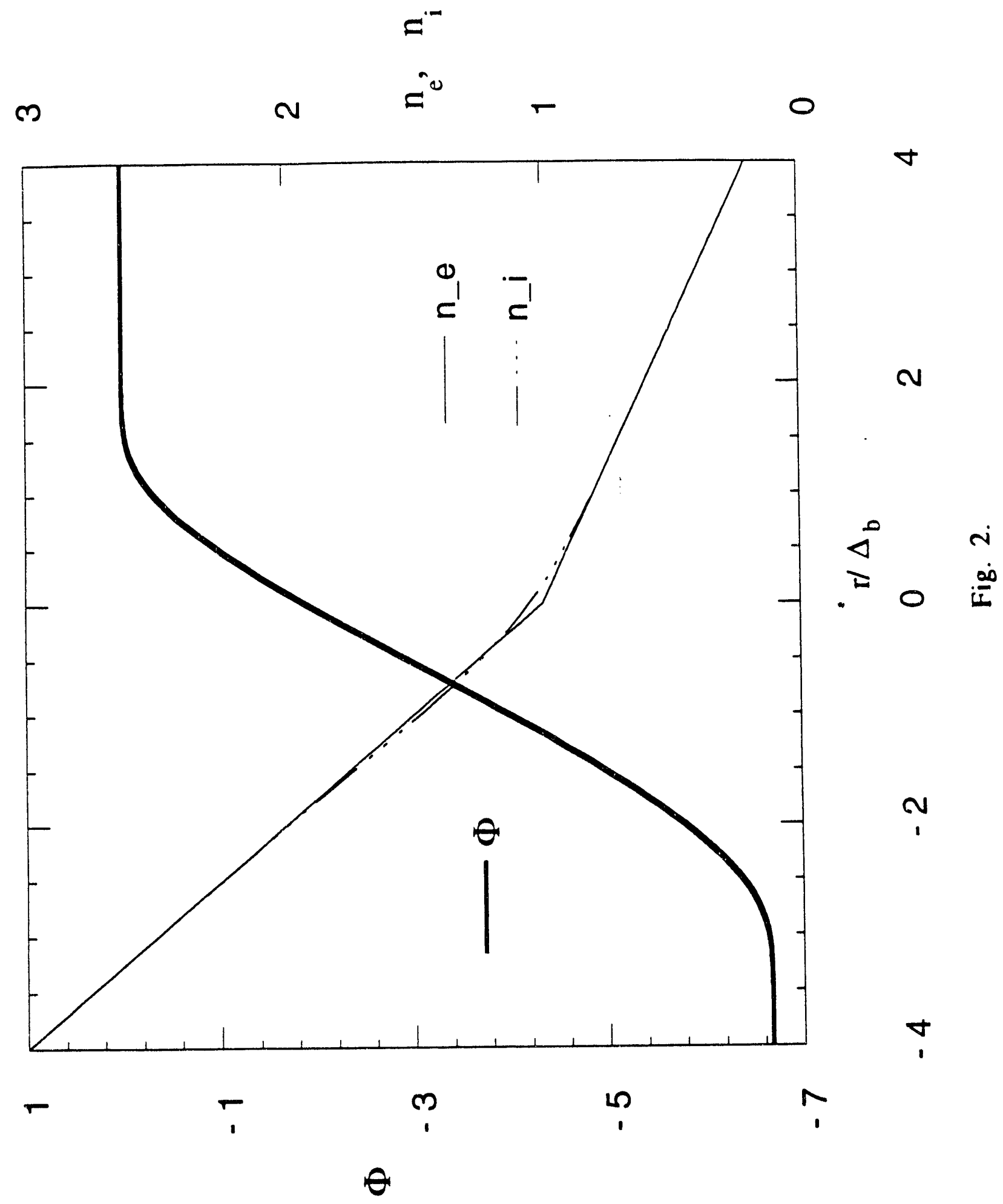



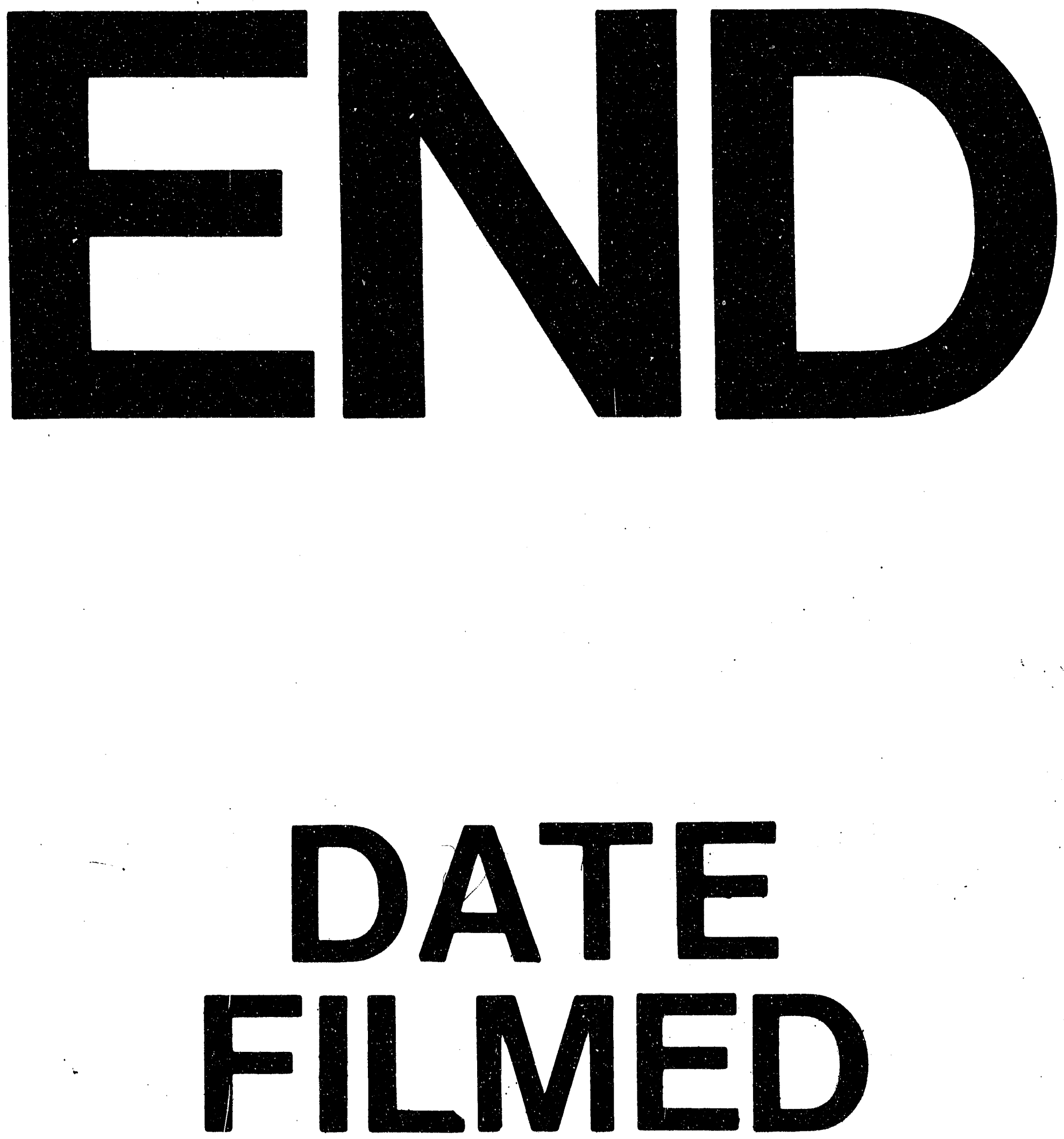

|

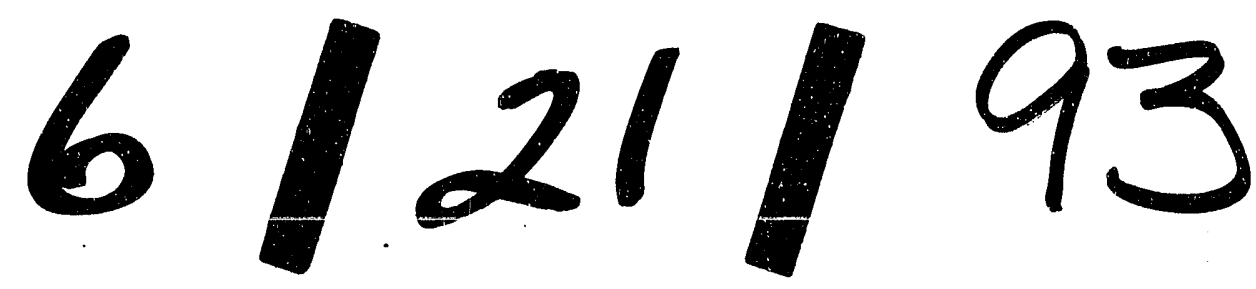


